Check for updates

Cite this: RSC Adv., 2019, 9, 19197

\title{
Enhanced inertial focusing of microparticles and cells by integrating trapezoidal microchambers in spiral microfluidic channels $\dagger$
}

\author{
Ala'aldeen Al-Halhouli, (D) *a Ahmed Albagdady, (D) ${ }^{a}$ Wisam Al-Faqheri, ${ }^{\text {b }}$ \\ Jonathan Kottmeier, ${ }^{c}$ Sven Meinen, (DD ${ }^{c}$ Lasse Jannis Frey, ${ }^{d}$ Rainer Krull ${ }^{d}$ \\ and Andreas Dietzel (D) ${ }^{c}$
}

In this work, manipulating width and equilibrium position of fluorescent microparticles in spiral microchannel fractionation devices by embedding microchambers along the last turn of a spiral is reported. Microchambers with different shapes and sizes were tested at Reynolds numbers between 15.7 and $156.6\left(100-1000 \mu \mathrm{L} \mathrm{min}^{-1}\right.$ ) to observe focusing of 2, 5 and $10 \mu \mathrm{m}$ fluorescent microparticles. This paper also discusses the fabrication process of the microfluidic chips with femtosecond laser ablation on glass wafers, as well as a particle imaging velocimetry ( $\mu \mathrm{PIV}$ ) study of microparticle trajectories inside a microchamber. It could be demonstrated with an improved final design with inclined microchamber side walls, that the $2 \mu \mathrm{m}$ particle equilibrium position is shifted towards the inner wall by $\sim 27 \mu \mathrm{m}$ and the focusing line's width is reduced by $\sim 18 \mu \mathrm{m}$. Finally, Saccharomyces cerevisiae yeast cells were tested in the final chip and a cell focusing efficiency of $99.1 \%$ is achieved.

Received 13th May 2019

Accepted 12th June 2019

DOI: $10.1039 / c 9 r a 03587 g$

rsc.li/rsc-advances

particle separation, some of them are active; which rely on

\section{Introduction}

Microparticle and cell sorting are of great research interest ${ }^{1-6}$ and promise applications in many different fields, such as separating circulating tumour cells from blood cells, ${ }^{7-9}$ and stem cell research. ${ }^{10}$ Microfluidics also includes applications in various emerging disciplines, such as biologically-inspired colouring, ${ }^{11}$ concentration detection, ${ }^{12}$ flexible and stretchable sensors ${ }^{13,14}$ and liquid crystal actuators. ${ }^{15}$ Microparticle focusing refers to the sorting of fluorescent microparticles or cells inside microfluidic channels under inertial flow conditions. When two or more microparticle types are focused inside a channel at different positions, it becomes possible to separate them in high throughput and purity. The focusing position of the microparticles depends mainly on the medium fluid's properties and flow rates, as well as particle diameter, particle shape, and channel geometries. In biological and biomedical research, isolating certain cells from a heterogeneous background is a key tool for studying selected rare species of cells and particles. ${ }^{16}$ Various microfluidic methods were developed to achieve high-efficiency

\footnotetext{
${ }^{a}$ NanoLab, School of Applied Technical Sciences, German Jordanian University, Amman, Jordan. E-mail: alaaldeen.alhalhouli@gju.edu.jo

${ }^{b}$ MicroNano Mechatronic Lab, Mechanical, Automotive \& Materials Engineering, University of Windsor, Windsor, ON, Canada

'Institut für Mikrotechnik, Technische Universität Braunschweig, Braunschweig, Germany

${ }^{d}$ Zentrum für Pharmaverfahrenstechnik, Technische Universität Braunschweig, Braunschweig, Germany

$\dagger$ Electronic supplementary information (ESI) available. See DOI: $10.1039 / \mathrm{c} 9 \mathrm{ra} 03587 \mathrm{~g}$
} external forces to manipulate microparticles such as magnetic, ${ }^{17,18}$ dielectrophoretic (DEP) ${ }^{19}$ optic, ${ }^{20}$ and cell sorting with flow cytometry, ${ }^{21}$ while others rely only on hydrodynamic forces as pinched flow fractionation (PFF) ${ }^{22}$ Deterministic lateral displacement (DLD) ${ }^{23}$ microvortex manipulation, ${ }^{24}$ inertial and dean flow fractionation..$^{25-29}$ Passive focusing does not require complex setup and exhibits high throughput, portability, and purity. ${ }^{16}$ In the passive technique known as inertial microfluidics, microparticle focusing relies only on hydrodynamic forces inside the microchannels that affect microparticles and let them migrate towards their equilibrium positions..$^{30,31} \mathrm{~A}$ multi-turn spiral microchannels device for inertial focusing uses curved channels to develop a secondary flow that induces a Dean force $\left(F_{\mathrm{D}}\right)$ which balances with existing lift forces, both forces act on the microparticles in different directions and magnitudes. ${ }^{8,32}$ Consequently, relatively large particles have the tendency to migrate towards the inner channel wall due to the high lift they experience while smaller particles migrate towards the channel's outer wall. ${ }^{16}$ Spiral microfluidic chips are well studied in the literature and different designs were established and evaluated. Curved channels induce a Dean flow ${ }^{33,34}$ that appears as two counter-rotating vortices located in the lower and upper halves of the channel cross section. ${ }^{35-37}$ Nivedita et al. ${ }^{32}$ described the essential forces and fluid dynamics involved in microparticles focusing inside a Dean flow, in reference to Dean's original work in $1928,{ }^{38}$ they stated that the maximum velocity point shifts from the centre towards the outer (concave) wall inducing a sharp velocity profile that increases pressure leading to a fluid recirculation known as Dean vortices. The Dean force acting on particles depends mainly 
on particle diameter and flow velocity and becomes dominant to inertial forces in smaller particle diameters. ${ }^{26}$ Another method to induce a secondary flow (Dean flow) is by implementing a contraction-expansion array (CEA), ${ }^{39}$ where accompanied by Dean flow the streamlines closer to the expansion region experience acceleration and follow a curved path, therefore inducing a Dean flow. Channel expansions have been reported ${ }^{\mathbf{4 0 - 4 2}}$ as a method of isolation and trapping of cells. In another study ${ }^{\mathbf{4 3}} \mathrm{A}$ threshold Reynold's number was found for each particle size, at which, microparticles will be trapped inside a laminar microvortex. As the desired particle size decreases, the forces affecting it decreases drastically as Cruz et $a .^{44}$ described when aiming to focus particles as small as $1 \mu \mathrm{m}$. Therefore, when aiming to focus smaller particles (less than $5 \mu \mathrm{m}$ ), this force reduction has to be compensated by increasing the flow rate or by decreasing the channel dimensions. However, this might not be easily realizable as it requires operation with higher pressure drops over the microchannels, inducing problems with leakages or pump stalling. The method of improving microparticle focusing even at lower flow rates is to integrate microchambers along the last turn of the spiral channel that changes the parabolic velocity profile and force the particles to converge to a smaller region. As a result, focusing microparticles should be significantly improved in terms of focusing width and distance from the inner wall under lower flow rate values. The effectiveness of microfluidic fractionation devices is usually tested with polystyrene fluorescent microparticles or PDMS (polydimethylsiloxane) beads; ${ }^{45}$ however, the main application of these devices is often to filter, isolate or label biological samples. ${ }^{46}$ These samples are not identical to polystyrene fluorescent microparticles in terms of diameter consistency or shape, therefore, it is essential to design a chip that is robust to small variance in the targeted cell morphology. Warkiani et al. reported CTC (circulating tumour cells) isolation in spiral microfluidic chips with and without sheath fluid. ${ }^{\mathbf{8} 36}$ Baker yeast Saccharomyces cerevisiae $(\varnothing 5-10 \mu \mathrm{m})$ shall be used to test focusing robustness for biological objects which are deformable. $\mu \mathrm{PIV}$ as a non-contact method using fluorescent tracer particles shall be used to obtain the flow vector field in twodimensions. Using two cameras in a stereoscopic setup, even 3-D components of the flow vectors can be obtained. ${ }^{47-49}$ In order to detect fluid's motion using $\mu \mathrm{PIV}$, fluorescent tracer particles are added and two sequential images are captured and analysed using spatial correlation methods that calculate the fluid velocity fields from the motion of the tracer particles over a known time interval. ${ }^{50}$ This technique has recently been used to investigate vortex formation in glass micro bioreactors under the influence of capillary waves. ${ }^{\mathbf{5 1}}$ This technique shall be utilized to analyse and observe flow velocity profiles inside the Dean flow inducing microchambers to gain a better perspective on the local flow conditions that shall lead to focusing enhancement as long as the tracer particles (smaller than the particles that experience fractionation) are following the flow and are unaffected by inertia. The software calculates a cross-correlation of intensities within an interrogation window, a sequence of usable images needs a high seeding density. The $\mu$ PIV study shall be conducted on isosceles trapezoidal microchambers to experimentally investigate the changes in velocity profile leading to enhanced focusing.

\section{Microfluidic chip design and methodology}

Developed chips consist of an 8-turn spiral with one inlet at the chip edge and two outlets in the centre of the chip (see Fig. 1). The channel was designed with $200 \mu \mathrm{m}$ width, $50 \mu \mathrm{m}$ depth with 19 microchambers along the last turn of the spiral path. Embedded microchambers are fabricated in different widths, heights, shapes as explained in Table 1 and referenced in Fig. 1(c) to study the effect that these parameters induce on the focusing trajectory whether the effect is shifting the stream along the channel width or changing the width of the particles equilibrium position. Also, they are tested with 2,5 and $10 \mu \mathrm{m}$ particles.

At first, a spiral channel without microchambers was fabricated as a reference to compare the results acquired in each microchamber design. The wall and shear gradient lift forces act against each other to eventually settle the particles in an equilibrium position, the wall-induced lift force $F_{\mathrm{w}}$ depends on the distance from the wall $\delta$ with $F_{\mathrm{w}} \propto a^{3} / \delta$ (ref. 52) and practically vanishes when a particle enters a microchamber. ${ }^{53}$ Microchambers are designed with inclined side walls to reduce particles' vertical velocity component as they exit the microchamber. Fig. 2 illustrates the influence of inclined microchamber's side walls on the particles' exit angle $\theta_{\mathrm{P}}$ and on gradual decay and recovery of wall lift force by comparing a rectangular microchamber (Fig. 2(a)) and a trapezoidal one (Fig. 2(b)). As will be discussed in a following section, the inclined side walls play a decisive role in microparticle focusing, because, at a given flow rate, microparticle's exit angle with perpendicular walls is lower than with inclined walls.

\section{Microfluidic chip fabrication}

Engraving glass to fabricate the microfluidic channels is an iterative process in optimizing the femtosecond laser parameters to achieve the desired depth and width as well as to result in a suitable surface finish as has been investigated in earlier works. ${ }^{54,55}$ Also, a detailed process for trapezoidal channels was introduced $^{25}$ with adjusted laser filling strategies. Once the glass wafer is engraved, it is treated in an HF-based glass etching solution ( $45 \mathrm{~mL} \mathrm{H} \mathrm{H}_{2} \mathrm{O}, 100 \mathrm{~mL} \mathrm{H}_{3} \mathrm{PO}_{4}$, and $30 \mathrm{~mL} \mathrm{HF}$ ) for $90 \mathrm{~s}$ to smoothen the engraved surface and get rid of any residual glass fragments. The wafer is then cleaned with a wafer cleaning machine (Fairchild Convac, Neuenstadt, Germany) and flooded with a mixture of $\mathrm{H}_{2} \mathrm{SO}_{4}$. To seal the chips, another blank glass wafer is bonded on the top and left in a muffle oven at $620^{\circ} \mathrm{C}$ for 6 hours for bonding (see Fig. 3). Fig. 4(a) shows a 3D microscope image (Keyence VHX 5000, Japan-imported through Keyence Germany) of a channel's cross-section between two microchambers. The laser filling strategies are demonstrated in Fig. 4(b) and are further refined based on the previous work, ${ }^{25}$ to allow for new channel features and calibration with actual laser beam performance. A set of intersecting parallel lines (0, 90, 45, and $135^{\circ}$ ) are used to cover the channel's area and get it to the required depth. The shape resulting from this strategy exhibits inclined walls instead of perpendicular, therefore equidistant contour lines positioned 4 and $25 \mu \mathrm{m}$ away from the walls are added to straighten the side walls. 


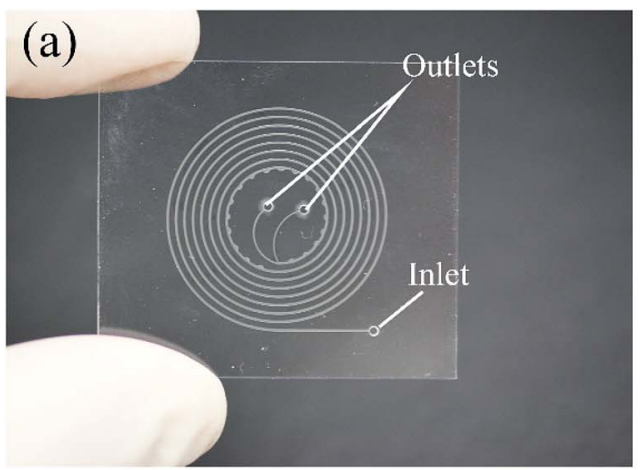

(b)

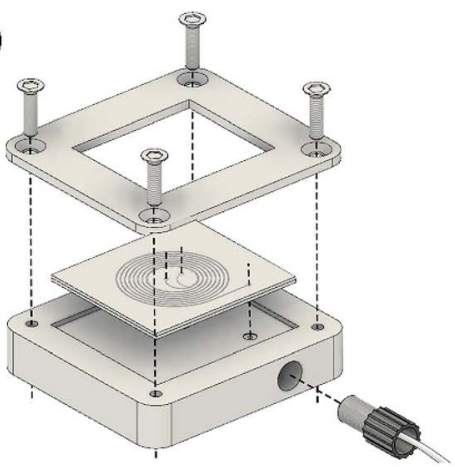

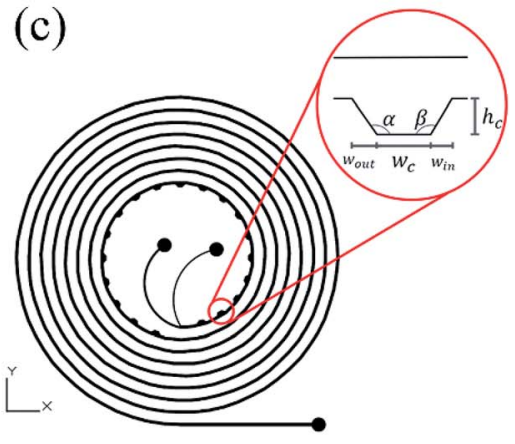

Fig. 1 (a) Investigated microfluidic chip with two outlets and one inlet. In this design, the microchambers are positioned at the inner wall of the channel, (b) 3D illustration of the aluminium adapter used to fix the chip for easier tubes connections, (c) microfluidic chip design showing standard variables of microchambers design, $W_{c}$ stands for the width of the flat region in the microchamber, $W_{\text {in }}$ is the width of the microchamber's entry wall, $W_{\text {out }}$ is the width of the microchamber's exit wall, $h_{\mathrm{c}}$ is the microchamber's height, $\alpha$ is the exit wall's angle and $\beta$ is the entry wall angle.

Table 1 Types and dimensions of all fabricated microchambers that were integrated into spiral microfluidic channels. The rectangular type was the design that was tested at first. Refer to Fig. 1(c) for variables representation

\begin{tabular}{llllrr}
\hline Microchamber type & $W_{\mathrm{c}}[\mu \mathrm{m}]$ & $W_{\text {in }}[\mu \mathrm{m}]$ & $W_{\text {out }}[\mu \mathrm{m}]$ & $h_{\mathrm{c}}[\mu \mathrm{m}]$ & $\alpha\left[^{\circ}\right]$ \\
\hline Rectangular & 300 & 0 & 0 & 200 & 90 \\
Square & 300 & 0 & 0 & 300 & 90 \\
Wide rectangular & 500 & 0 & 0 & 200 & 90 \\
Right trapezoidal & 300 & 0 & 200 & 200 & 90 \\
Wide right trapezoidal & 500 & 0 & 200 & 200 & 135 \\
Isosceles trapezoidal & 300 & 200 & 200 & 200 & 90 \\
& & & & 90 \\
\end{tabular}

The surface finish of the channels is crucial since small particles might get trapped inside the micro-crevices obstructing the monitoring process and creating undesired artefacts, Fig. 5 shows a surface roughness profile measured over $677 \mu \mathrm{m}$ of the channel bottom after two different fabrication steps along with their corresponding values of $R_{\mathrm{a}}$ (average roughness) and $R_{\mathrm{z}}$ (the difference between highest and lowest peaks). Fig. 5(a) shows a measurement obtained directly after laser ablation showing a rough surface with sharp edges, while Fig. 5(b) shows an improved roughness profile obtained after HF glass etching

a)

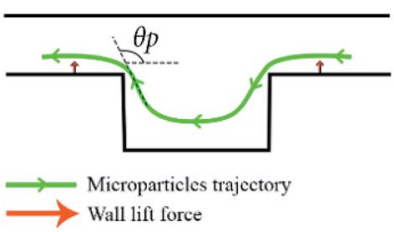

b)

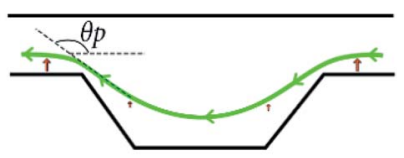

Fig. 2 Schematic illustration of microchamber's effect on microparticle focusing with a demonstration of wall lift force decay as the particle enters the microchamber and microchamber shape influence on particle exit angle $\theta_{\mathrm{p}}$, (a) particles trajectory for rectangular microchamber type, the particles would experience a sudden change in wall lift force as they enter the chamber and when they depart with $\theta_{\mathrm{P}}$ close to $90^{\circ}$, (b) particles trajectory for isosceles trapezoidal microchamber type, the particles would experience a smooth decay in wall lift force as they enter the chamber and a smooth recovery when they depart it with $\theta_{\mathrm{P}}$ close to $135^{\circ}$. because the acid breaks the siloxane bonds in the silicate network ${ }^{56}$ preferentially attacking sharp geometries and leaving behind a smoothed surface.

\section{$\mu$ PIV setup}

The setup is based on a V20 stereomicroscope equipped with two Imager sCMOS cameras at $50 \mathrm{~Hz}$ illuminated by a Litron Bernoulli Nd:YAG laser at an excitation wavelength of $523 \mathrm{~nm}$ (FlowMaster PIV, LaVision, Göttingen Germany). To allow a constant seeding density without being affected by the focusing enhancement, $1.5 \mu \mathrm{m}$ red fluorescent spherical polystyrene microparticles were used for tracking (exc./emm.: 532/ 605, PS FluoRot, Microparticles GmbH, Berlin, Germany), using both cameras in stereoscopic mode allows the observation of the out-of-plane movement. DaVis 8 (LaVision, Göttingen Germany) software was used to analyse the captured images and calculate the velocity profile.

\section{Saccharomyces cerevisiae cells preparation}

The Saccharomyces cerevisiae N34 strain was stored at $-80{ }^{\circ} \mathrm{C}$ in $30 \%(\mathrm{v} / \mathrm{v})$ Glycerin with lysogeny broth medium. Inocula were grown overnight in $250 \mathrm{~mL}$ shaken flasks (Schott, Mainz, Germany; $20 \%(\mathrm{v} / \mathrm{v})$ filled at $30{ }^{\circ} \mathrm{C}$ and $\left.120 \mathrm{~min}^{-1}\right)$ and diluted to an OD of 0.1 (SmartSpec 3000 Spectrophotometer, Bio-Rad Laboratories, München, Germany). For inducing a specific fluorescence response, previously shown in ref. 55, the culture broth was used to investigate the controlled initiation of oxidative 


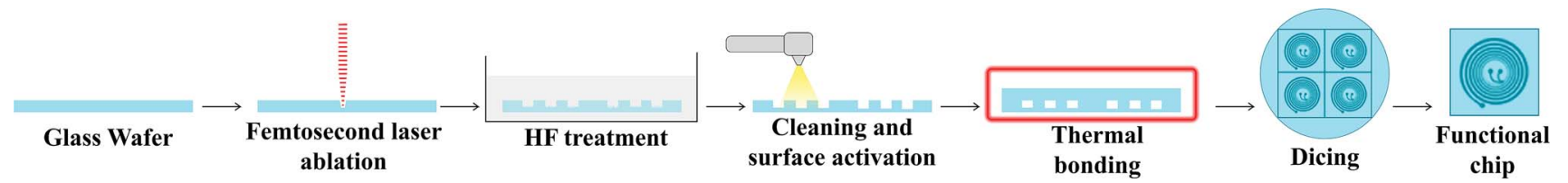

Fig. 3 Fabrication steps for glass microfluidic chips. $700 \mu \mathrm{m}$ thick glass wafers are engraved using the femtosecond laser machine to create four microfluidic chip structures. Subsequently, wafers are HF treated and cleaned carefully before sealing by thermal bonding. Finally, the wafer is diced to separate four chips.

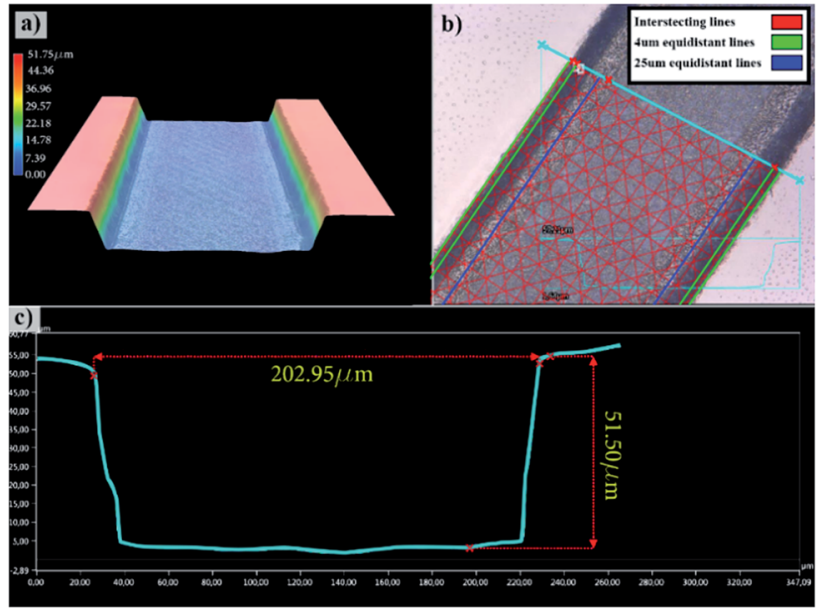

Fig. 4 3D microscope images of the fabricated microfluidic chip, (a) 3D representation of the channel, (b) channel with overlaid with the used femtosecond laser contour line pattern, (c) optically-measured channel's cross-sectional profile.

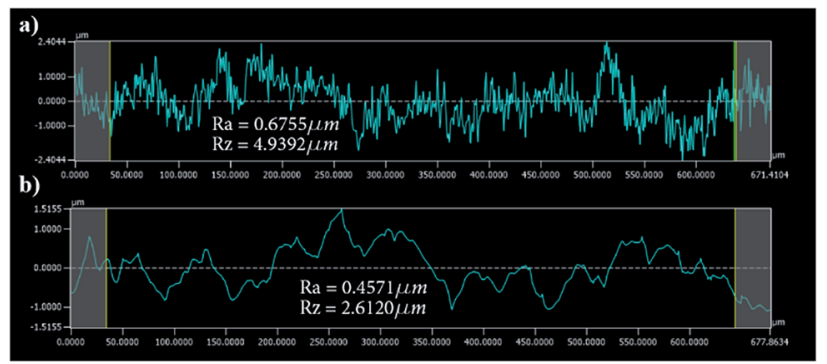

Fig. 5 Surface roughness results over a $677 \mu \mathrm{m}$ of the channel surface (a) roughness results after laser ablation (b) after HF etching.

stress in yeast cells. Therefore, $5 \mathrm{~mL}$ of diamide (Sigma-Aldrich, Steinheim, Germany) with a load of $200 \mathrm{mg}$ diamide/g CDW (cell dry weight) were added to the culture broth.

\section{Results and discussion}

A set of experiments were performed on the fabricated spiral chips using 2, 5 and $10 \mu \mathrm{m}$ spherical polystyrene greenfluorescent microparticles with an excitation wavelength of $468 \mathrm{~nm}$ and an emission of $505 \mathrm{~nm}$ (Fluoro-Max, Thermo Fisher Scientific, Massachusetts, USA) and Saccharomyces cerevisiae yeast cells. $50 \mu \mathrm{L}$ of particles solution (in case of $1 \%(\mathrm{w} / \mathrm{v})$ fluorescent particle suspension) are diluted in $10 \mathrm{~mL}$ of deionized water, resulting in $0.0005 \%(\mathrm{w} / \mathrm{v})$ suspension. The mixture is then filled into a glass syringe, loaded into a syringe pump (NEMSYS Low-pressure module $14: 1$, Cetoni GmbH, Korbussen, Germany) and pumped with a wide range of flow rates $\left(100-1000 \mu \mathrm{L} \mathrm{min}{ }^{-1}\right)$. The experiments are monitored with a camera mounted on an inverted microscope (Leica DM IL LED) equipped with a blue LED light source (Leica SFL 100, both Leica Microsystems GmbH, Wetzlar, Germany).

\section{Microparticle focusing}

Prior to experiments, the chip was mounted on the inverted microscope and pumped with DI water for a few minutes to remove residuals and air bubbles. The pump is controlled from computer software (QmixElements, Cetoni $\mathrm{GmbH}$, Korbussen, Germany) that allows full control over pumped volume and flow rate as well as the syringe fill level. Fig. 6 shows the experimental results obtained with variations of the microchamber profile. Fig. 6(a) shows the influence of different microchamber designs (refer to Table 1) on focused line's width and location with respect to the inner wall. The flow rates at which images were taken were individually chosen for best focusing condition in terms of width and distance from the inner wall. Wide rectangular and square microchambers did not show improvement when compared to the initial rectangular type. The isosceles trapezoidal design shows a focusing line shift by $27 \mu \mathrm{m}$ towards the inner wall compared to the design without microchambers. In addition, the focusing line's width is decreased by $18 \mu \mathrm{m}$ as illustrated in Fig. 6(b).

The fluorescence light intensity across the channel's width is extracted for multiple microchamber's designs at the same flowrates mentioned in Fig. 6(a). The comparison between the designs is carried out on the number of equilibrium positions, standard deviation and mean value of the fluorescence light intensity across the channel (see Fig. 7), where the mean correlates to the average distance from the inner wall and the standard deviation correlates to the focusing line width. With one $135^{\circ}$ inclined edge the mean is shifted by $7 \mu \mathrm{m}$ towards the inner wall, while with both inclined edges no further shift on the mean was noted but a reduction of the standard deviation by $12.6 \mu \mathrm{m}$ was observed.

When particles enter the microchamber, the lack of wall lift force would pull the particles further down closer to the bottom of the chamber. In the right trapezoidal microchamber, the lowest point of the particle trajectory is above the inclined side wall rather than in its centre, this behaviour subjects the microparticles to the sudden onset of wall-lift force and elevates 
a)
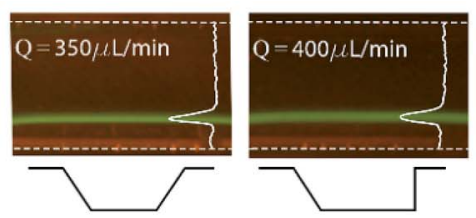

Isosceles trapezoidal Wide right trapezoidal Right trapezoidal

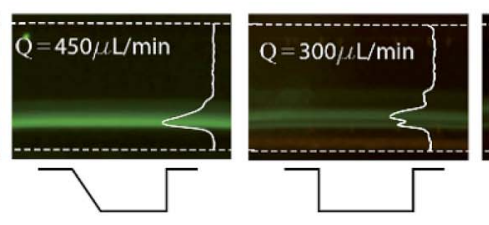

Wide rectangular

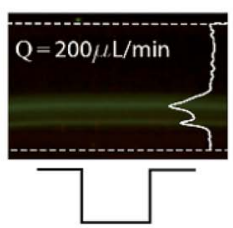

Square

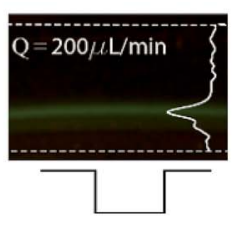

Rectangular

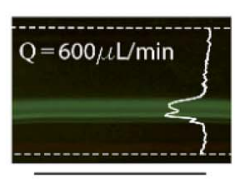

Without

b)
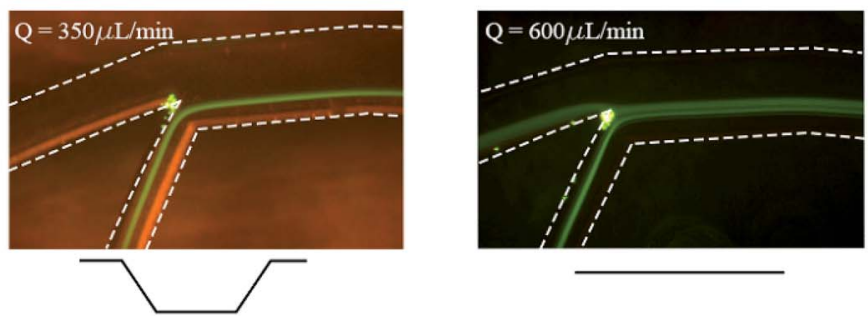

Fig. 6 Focused stream of $2 \mu \mathrm{m}$ fluorescent particles for various types of microchambers each taken at the flow rate that showed the best focusing result in terms of width and distance from the inner wall of the particles streamline All designs were fabricated with the same spiral channel dimensions. (a) Microparticle trajectories as observed at the end of the spiral just before the branch, with overlaid green colour intensity distribution across the channel's width. (b) Trajectories observed at the spiral's branch for a channel with no microchambers and a channel with isosceles trapezoidal microchambers.

their position. That issue does neither appear in wide right trapezoidal nor in the isosceles microchambers because these chambers are wide enough to keep the lowest point of particles stream further away from the inclined exit side wall. All microchamber designs did not disrupt the already existing focusing of microparticles but rather showed an improvement in particle focusing in comparison to spiral microchannel chips without microchambers.

5 and $10 \mu \mathrm{m}$ particles focused at positions close to the inner spiral microchannel wall in all fabricated chips for flow rates between 200 and $700 \mu \mathrm{L} \mathrm{min}^{-1}$. Since the height over width $(h /$ $w$ ) ratio is lower than 1 in the main channel, the velocity profile gets flattened, therefore, the shear gradient lift forces decrease ${ }^{57}$ causing the wall lift force to become more dominant leading to microparticles migration towards channel's centre as in the channel without microchambers design (refer to Fig. 6). One method to exert more force on microparticles is to increase the flowrate, however, higher Reynold's numbers changes the shape

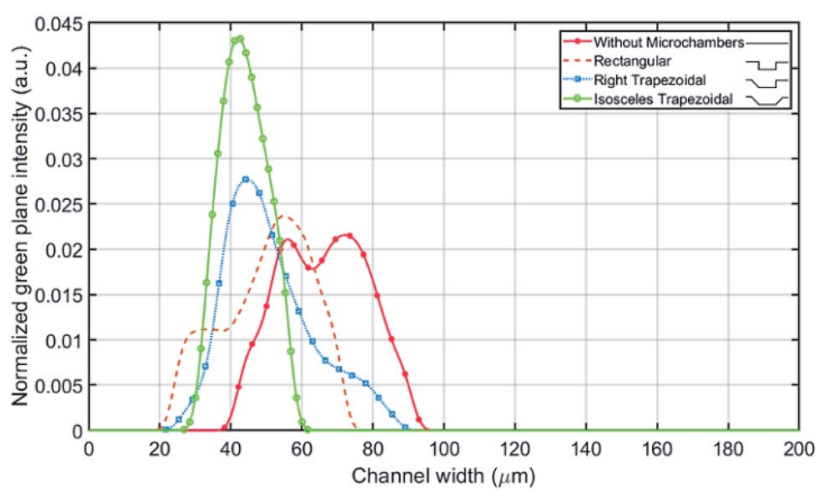

Fig. 7 Fluorescent light intensity profile for multiple microchambers types against the channel's width. of the velocity profile inside the microchannel, ${ }^{58}$ causing particles to focus in multiple equilibrium positions as seen in the design without microchambers. A $\mu$-PIV experiment was conducted on the isosceles trapezoidal microchamber to extract the actual velocity profile just before, after and inside the microchamber. Fig. 8 shows the $\mu$ PIV results acquired from inserting $1.5 \mu \mathrm{m}$ red fluorescent spherical polystyrene microparticles (exc./emm.: 532/605, PS FluoRot, Microparticles GmbH, Berlin, Germany). To gain reliable results from $\mu$ PIV, the particle's tracing fidelity should be quantified by Stokes number: ${ }^{59}$

$$
\mathrm{Stk}=\frac{\rho_{\mathrm{p}} d_{\mathrm{p}} V}{18 \mu d_{\mathrm{c}}}
$$

where $\rho_{\mathrm{p}}$ is the particle density, $d_{\mathrm{p}}$ is the particle diameter, $V$ is the fluid velocity, $\mu$ is the fluid dynamic viscosity and $d_{\mathrm{c}}$ is the characteristic dimension. The calculations returned a rather small Stokes number of 0.0011 for the $1.5 \mu \mathrm{m}$ particles. However, it is common in inertial microfluidic flows to have a small Stokes number, ${ }^{60}$ making it an indecisive measure of particle fidelity inside microchannels and the measured vector field is the particle's velocity field rather than the fluid's. The black streamlines in Fig. 8(a) indicate the paths particles would follow when exposed to the calculated vector field, the streamlines are a bit tighter at the microchamber's exit in comparison with at the entry, which indicates that inertial focusing is happening to the $1.5 \mu \mathrm{m}$ particles used to derive the velocity field. Sections A and C in Fig. 8(a) shows the particles velocity profile inside a microchannel between two microchambers, the velocity profile in that area is skewed towards the inner wall, and consequently increasing the shear gradient lift force between the inner wall and the maximum velocity point (coded in dark red). Sections B and D show the velocity profile inside the microchamber, the decrease in velocity is mainly because of the width expansion, however, the velocity in section B is 
a)
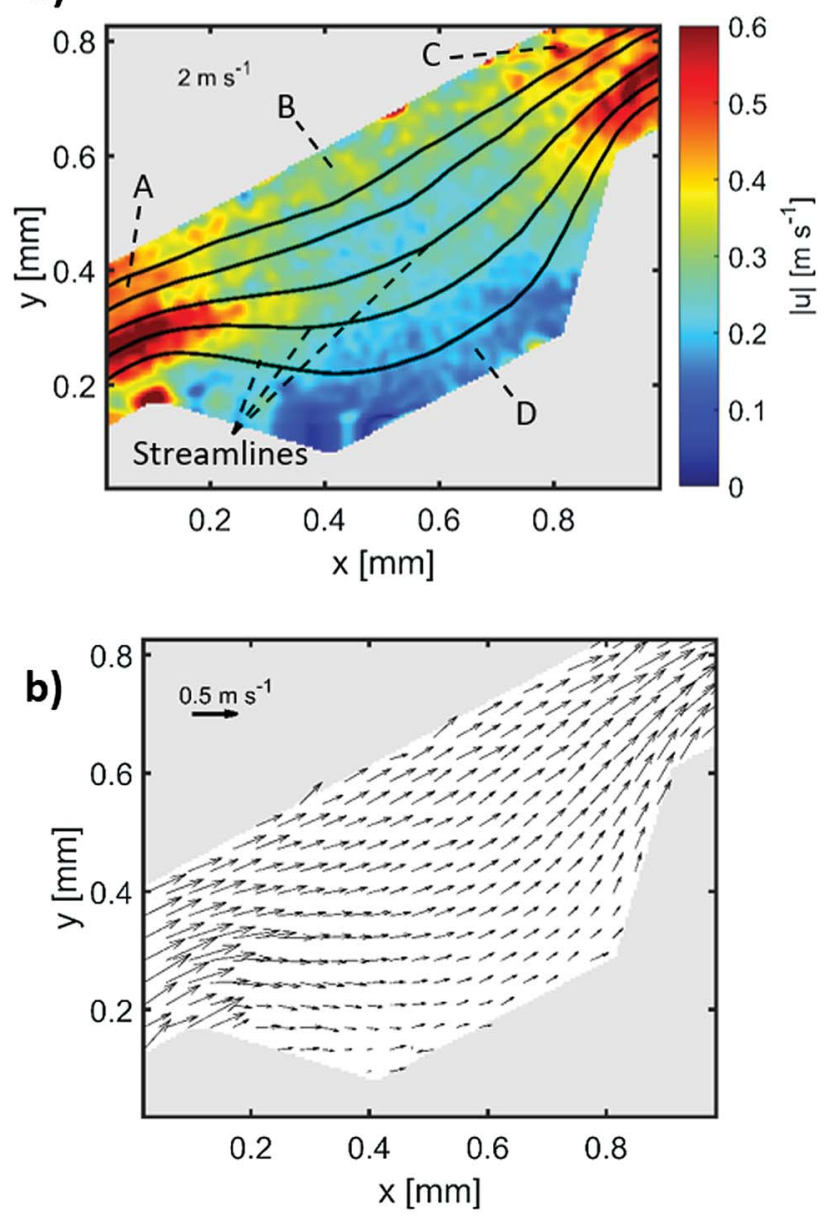

Fig. $8 \mu \mathrm{PIV}$ results inside an isosceles trapezoidal microchamber, (a) velocity profile inside the microchamber, (b) flow direction inside the last microchamber

slightly higher than section $\mathrm{D}$ because the fluid in that area travels a longer distance. Fig. 8(b) shows the velocity arrows to indicate the flow's direction and magnitude.

\section{Yeast cells focusing}

The microfluidic chip with embedded microchambers was tested on Saccharomyces cerevisiae cells (size $5-10 \mu \mathrm{m}$ ). The cells were expected to focus near the inner wall since particles 2,5 and $10 \mu \mathrm{m}$ focused in that area. In this experiment, the concentration of cells can be increased because they would focus and exit through one outlet only. The cultivation broth was diluted in $10: 1$ ratio (DI water : cells solution) and pumped into the isosceles trapezoidal chip with a flow rate of 350 $\mu \mathrm{L}$ min $^{-1}$ which could focus 2,5 and $10 \mu \mathrm{m}$ microparticles near the inner wall. Cells were either in pre-replicative phases $\left(\mathrm{G}_{0}\right.$ and $\mathrm{G}_{1}$ ) or DNA replicating phase (S). ${ }^{61}$ In those phases, cells may have small emerging buds which are identified as having a radius less than $50 \%$ of the mother cell. ${ }^{62}$ This experiment shall test the robustness of the chip design to focus shapevarying $^{63}$ yeast cells. Haemocytometer results gave an initial concentration of $164.5 \times 10^{4}$ cells per $\mathrm{mL}$ before the experiment, $306.5 \times 10^{4}$ cells per $\mathrm{mL}$ on the inner wall outlet and 2.75

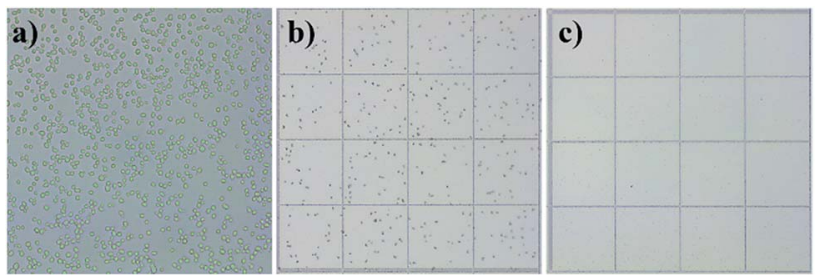

Fig. 9 Cell distributions as observed in microscopic images at different experiment steps, (a) undiluted sample before the experiment taken with a $40 \times$ objective zoom lens showing cells morphology, (b) inner wall outlet fluid sample from one haemocytometer square showing high cell concentration, (c) outer wall outlet fluid sample from one haemocytometer square showing low cell concentration.

$\times 10^{4}$ cells per $\mathrm{mL}$ on the outer wall outlet, therefore, a $99.1 \%$ focusing efficiency was achieved. This implies an increase in concentration by $186.3 \%$ at the inner wall outlet and a decrease by $598.1 \%$ at the outer wall outlet. Fig. 9(a) shows cell morphology in an undiluted cells culture before the experiment. Most cells are single cells, but a small percentage started budding. Outlet fluids are examined in a haemocytometer showing high cell concentration from the inner wall outlet (Fig. 9(b)), and extremely low cells concentration from the outer wall outlet (Fig. 9(c)). The focusing efficiency is calculated as $99.1 \%$ by dividing cells concentration at the inner wall by the total concentration from both outlets.

\section{Conclusions}

Trapezoidal microchambers have been shown to greatly enhance focusing of small microparticles $(2 \mu \mathrm{m})$ by changing the velocity profile inside the main channel, which directly correlates to an increase in shear gradient lift force, therefore shifting the microparticles by $\sim 27 \mu \mathrm{m}$ towards the inner wall, reducing the focusing line's width by $\sim 18 \mu \mathrm{m}$ also reducing the number of equilibrium positions in the proposed isosceles trapezoidal design. Different microchambers shapes were introduced and evaluated to study the behaviour of microparticles inside spiral channels with microchambers. The inclined microchambers sidewalls prevent particles from overshooting towards the outer wall as they exit the chamber and the skewed velocity profile exerts shear gradient lift force towards the inner wall, these conclusions were supported by a $\mu$ PIV study that experimentally extracts the fluid's velocity profile. Comparing to other works, ${ }^{2,8,36}$ an ultra-thin stream of particles was achieved and smaller particle size could be focused, therefore, the fabricated chip is ideal for particle filtration applications as high-efficiency blood plasma extraction. ${ }^{64}$ Saccharomyces cerevisiae cells were tested and successfully focused with 99.1\% efficiency to check the focusing robustness on biological samples paving the way towards cells sorting and enrichment.

\section{Conflicts of interest}

There are no conflicts to declare. 


\section{Acknowledgements}

This research is financially supported by the German Academic Exchange Service (DAAD) under the project entitled "Inertial focusing for continuous nanoparticles separation in femtosecond laser 3D micromachined curved channels”. Special thanks to Nanolab engineers, especially Zaid Doofesh for assisting in chips fabrication and Loiy Alghussain for his support in data analysis. Also, special thanks to Adnan AlLahham and Sundos Asfour for assisting in early cells experiments. Jonathan Kottmeier is funded by the DFG Project SPP2045 "MehrDimPart". Lasse Frey and Sven Meinen gratefully acknowledge financial support from the German Research Foundation (DFG) within the project "Development of microreactors for biopharmaceutical applications" (DI 1934/9-1, KR 1897/5-1).

\section{References}

1 T. Yang, P. Paiè, G. Nava, F. Bragheri, R. M. Vazquez, P. Minzioni, M. Veglione, M. Di Tano, C. Mondello, R. Osellame and I. Cristiani, Lab Chip, 2015, 15, 1262-1266. 2 M. Terashima, E. S. Freeman, R. E. Jinkerson and M. C. Jonikas, Plant J., 2015, 81, 147-159.

3 M. J. Tomlinson, S. Tomlinson, X. B. Yang and J. Kirkham, J. Tissue Eng., 2013, 4, 1-14.

4 L. Liu, T. H. Cheung, G. W. Charville and T. A. Rando, Nat. Protoc., 2015, 10, 1612-1624.

5 D. T. Pederick, K. L. Richards, S. G. Piltz, R. Kumar, S. Mincheva-Tasheva, S. A. Mandelstam, R. C. Dale, I. E. Scheffer, J. Gecz, S. Petrou, J. N. Hughes and P. Q. Thomas, Neuron, 2018, 97, 59-66.

6 P. M. Holloway, J. Butement, M. Hegde and J. West, Biomicrofluidics, 2018, 12, 044104.

7 P. Li, Z. S. Stratton, M. Dao, J. Ritz and T. J. Huang, Lab Chip, 2013, 13, 602-609.

8 M. E. Warkiani, B. L. Khoo, L. Wu, A. K. P. Tay, A. A. S. Bhagat, J. Han and C. T. Lim, Nat. Protoc., 2016, 11, 134-148.

9 B. Kwak, J. Lee, J. Lee, H. S. Kim, S. Kang and Y. Lee, Biosens. Bioelectron., 2018, 101, 311-316.

10 J. F. Zhong, Y. Chen, J. S. Marcus, A. Scherer, S. R. Quake, C. R. Taylor and L. P. Weiner, Lab Chip, 2007, 8, 68-74.

11 L. Shang, W. Zhang, K. Xu and Y. Zhao, Mater. Horiz., 2019, 6, 945-958.

12 J. Jeon, N. Choi, H. Chen, J. Il Moon, L. Chen and J. Choo, Lab Chip, 2019, 19, 674-681.

13 O. Y. Kweon, M. Y. Lee, T. Park, H. Jang, A. Jeong, M. K. Um and J. H. Oh, J. Mater. Chem. C, 2019, 7, 1525-1531.

14 D. Green Marques, P. Alhais Lopes, A. T. De Almeida, C. Majidi and M. Tavakoli, Lab Chip, 2019, 19, 897-906.

15 Y. Shang, J. Wang, T. Ikeda and L. Jiang, J. Mater. Chem. C, 2019, 7, 3413-3428.

16 W. Al-Faqheri, T. H. G. Thio, M. A. Qasaimeh, A. Dietzel, M. Madou and A. Al-Halhouli, Microfluid. Nanofluid., 2017, 21, 1-23.
17 S. Miltenyi, W. Müller, W. Weichel and A. Radbruch, Cytometry, 1990, 11, 231-238.

18 Q.-H. Gao, W.-M. Zhang, H.-X. Zou, W.-B. Li, H. Yan, Z.-K. Peng and G. Meng, Mater. Horiz., 2019, DOI: 10.1039/ C8MH01616J.

19 P. R. C. Gascoyne, X.-B. Wang, Y. Huang and F. F. Becker, IEEE Trans. Ind. Appl., 1997, 33, 670-678.

20 D. McGloin, Philos. Trans. R. Soc., A, 2006, 364, 3521-3537.

21 R. Krull, T. Wucherpfennig, M. E. Esfandabadi, R. Walisko, G. Melzer, D. C. Hempel, I. Kampen, A. Kwade and C. Wittmann, J. Biotechnol., 2013, 163, 112-123.

22 M. Yamada, M. Nakashima and M. Seki, Anal. Chem., 2004, 76, 5465-5471.

23 L. R. Huang, E. C. Cox, R. H. Austin and J. C. Sturm, Science, 2004, 304, 987-990.

24 C. H. Hsu, D. Di Carlo, C. Chen, D. Irimia and M. Toner, Lab Chip, 2008, 8, 2128-2134.

25 A. Al-Halhouli, W. Al-Faqheri, B. Alhamarneh, L. Hecht and A. Dietzel, Micromachines, 2018, 9, 171.

26 S. S. Kuntaegowdanahalli, A. A. S. Bhagat, G. Kumar and I. Papautsky, Lab Chip, 2009, 9, 2973-2980.

27 M. E. Warkiani, A. K. P. Tay, G. Guan and J. Han, Sci. Rep., 2015, 5, 1-10.

28 J. H. Lee, S. K. Lee, J. H. Kim and J. H. Park, Sens. Actuators, A, 2019, 286, 211-219.

29 R. Moloudi, S. Oh, C. Yang, M. Ebrahimi Warkiani and M. W. Naing, Microfluid. Nanofluid., 2018, 22, 1-14.

30 B. L. Khoo, M. E. Warkiani, D. S.-W. Tan, A. A. S. Bhagat, D. Irwin, D. P. Lau, A. S. T. Lim, K. H. Lim, S. S. Krisna, W.-T. Lim, Y. S. Yap, S. C. Lee, R. A. Soo, J. Han and C. T. Lim, PLoS One, 2014, 9, e99409.

31 A. A. S. Bhagat, S. S. Kuntaegowdanahalli and I. Papautsky, Phys. Fluids, 2008, 20, 1-4.

32 N. Nivedita, P. Ligrani and I. Papautsky, Sci. Rep., 2017, 7, 110.

33 T. Scherr, S. Pursley, W. T. Monroe and K. Nandakumar, Biomicrofluidics, 2013, 7, 1-15.

34 S. Sofela, S. Sahloul, M. Rafeie, T. Kwon, J. Han, M. E. Warkiani and Y. A. Song, Lab Chip, 2018, 18, 679-687.

35 N. Xiang, Z. Shi, W. Tang, D. Huang, X. Zhang and Z. Ni, RSC Adv., 2015, 5, 77264-77273.

36 M. E. Warkiani, G. Guan, K. B. Luan, W. C. Lee, A. A. S. Bhagat, P. Kant Chaudhuri, D. S. W. Tan, W. T. Lim, S. C. Lee, P. C. Y. Chen, C. T. Lim and J. Han, Lab Chip, 2014, 14, 128-137.

37 L. Balasubramaniam, R. Arayanarakool, S. D. Marshall, B. Li, P. S. Lee and P. C. Y. Chen, J. Micromech. Microeng., 2017, 27, 095016.

38 W. R. Dean, Proc. R. Soc. A, 1928, 121, 402-420.

39 M. G. Lee, S. Choi and J. K. Park, Appl. Phys. Lett., 2009, 95, 97-100.

40 E. Sollier, M. Cubizolles, Y. Fouillet and J. L. Achard, Biomed. Microdevices, 2010, 12, 485-497.

41 E. Sollier, M. Cubizolles, M. Faivre, Y. Fouillet and J. L. Achard, Proc. 31st Annu. Int. Conf. IEEE Eng. Med. Biol. Soc. Eng. Futur. Biomed. EMBC, 2009, vol. 2009, pp. 70307033. 
42 A. Karimi, S. Yazdi and A. M. Ardekani, Biomicrofluidics, 2013, 7, 021501.

43 J. Zhou, S. Kasper and I. Papautsky, Microfluid. Nanofluid., 2013, 15, 611-623.

44 J. Cruz, T. Graells, M. Walldén and K. Hjort, Lab Chip, 2019, 19, 1257-1266.

45 S. Wang, K. J. Sung, X. N. Lin and M. A. Burns, PLoS One, 2017, 12, 1-13.

46 Y. Chen, R. H. Austin and J. C. Sturm, Biomicrofluidics, 2017, 11, 054107.

47 F. Scarano and M. L. Riethmuller, Exp. Fluids, 1999, 26, 513523.

48 S. T. Wereley and C. D. Meinhart, Annu. Rev. Fluid. Mech., 2010, 42, 557-576.

49 R. Lindken, M. Rossi, S. Große and J. Westerweel, Lab Chip, 2009, 9, 2551-2567.

50 C. Mărculescu, A. Avram, C. Pârvulescu, M. Avram and C. M. Bălan, International Journal of Engineering, Mathematical and Physical Sciences, 2013, 6.0.

51 S. Meinen, L. J. Frey, R. Krull and A. Dietzel, Micromachines, 2019, 10, 284.

52 P. Stephen Williams, S. Lee and J. Calvin Giddings, Chem. Eng. Commun., 1994, 130, 143-166.
53 A. J. MacH, J. H. Kim, A. Arshi, S. C. Hur and D. Di Carlo, Lab Chip, 2011, 11, 2827-2834.

54 P. Erfle, J. Riewe, H. Bunjes and A. Dietzel, Microfluid. Nanofluid., 2017, 1-12.

55 P. Erfle, J. Riewe, H. Bunjes and A. Dietzel, Micromachines, 2019, 10, 220.

56 G. A. C. M. Spierings, J. Mater. Sci., 1993, 28, 6261-6273.

57 J. M. Martel and M. Toner, Phys. Fluids, 2012, 24, 032001.

58 E. Keinan, E. Ezra and Y. Nahmias, Appl. Phys. Lett., 2015, 107, 193507.

59 M. Zhang, M. Xu and D. L. S. Hung, Meas. Sci. Technol., 2014, 25, 095204.

60 H. Amini, W. Lee and D. Di Carlo, Lab Chip, 2014, 14, 27392761.

61 P. Delobel and C. Tesnière, PLoS One, 2014, 9, 1-8.

62 B. Yang Yu, C. Elbuken, C. L. Ren and J. P. Huissoon, J. Biomed. Opt., 2011, 16, 066008.

63 B. Y. Yu, C. Elbuken, C. Shen, J. P. Huissoon and C. L. Ren, Sci. Rep., 2018, 8, 1-12.

64 M. Rafeie, J. Zhang, M. Asadnia, W. Li and M. E. Warkiani, Lab Chip, 2016, 16, 2791-2802. 